\title{
Cardiac Myxofibrosarcoma
}

National Cancer Institute

\section{Source}

National Cancer Institute. Cardiac Myxofibrosarcoma. NCI Thesaurus. Code 147004.

A low-grade sarcoma that arises from the heart. It is composed of spindle or rounded cells in a myxoid stroma. The most common location is the left atrium. 